\title{
DECOUPLING OF LINEAR TIME-VARYING SYSTEMS WITH A BOND GRAPH APPROACH
}

\author{
Stefan Lichiardopol \\ Christophe Sueur \\ L.A.G.I.S., UMR 8146 CNRS \\ Ecole Centrale de Lille, Cité Scientifique, BP48 \\ 59651 Villeneuve d'Ascq Cedex, France \\ E-mail: stefan.lichiardopol@ec-lille.fr, christophe.sueur@ec-lille.fr
}

\begin{abstract}
The aim of this paper is to study the structure of the bond graph model for solving the input-output decoupling problem. First a graphical procedure is proposed for the analysis of the LTV models and then a technique for determining the decoupling matrices using the bond graph representation of the system is introduced.
\end{abstract}

\section{INTRODUCTION}

The input-output decoupling problem has received much attention since the first development proposed in (Falb and Wolovich 1967). This problem is usually decomposed in several steps such as: input-output decoupling study, control law calculus (decoupling matrices) and analysis of the stability property of the controlled model.

The first development was concerned with linear models from a state representation. An extension to the time-varying models was proposed in (Porter 1969) and later similar approaches to nonlinear models have been proposed.

In the 80's, new approaches gave new insight in this problem. These approaches were based on different representations, such as the transfer representation or the graphical representation (Commault and Dion 1982, Dion 1983).

In (Bertrand 1997), a bond graph approach was first proposed for linear models. The input-output decoupling problem was solved with the concept of causal paths and the control law expression was characterized with the joint application of the geometric approach and the graphical approach. The stability of the controlled model was then studied.

In this paper the study of decoupling problem for the linear time-varying models, using graphical approach provided by the bond graph model. This study is divided into two parts: the analysis of the system, which involves determining whether the system can be decoupled or not, and the synthesis of the decoupling law.
Our interest is primarily with the time-varying multivariate linear differential system in equation (1), where $I$ is an interval.

$$
\left\{\begin{array}{l}
\dot{x}(t)=A(t) x(t)+B(t) u(t) \\
y(t)=C(t) x(t)
\end{array}, t \in I\right.
$$

In the sequel we consider the system to be square, with $m$ inputs and $m$ outputs, therefore $u$ and $y$ are $m$-tuples. The state variable $x$ is an $n$-tuple and the matrices $A, B$, $C$ have the compatible dimensions. The decoupling is performed using the feedback law (2), where $w$ is an $m$ tuple which represents the new input of the system and again $F$ and $G$ are matrices of compatible dimensions.

$$
u(t)=F(t) x(t)+G(t) w(t), t \in I
$$

The plant is said to be decoupled if the $i^{\text {th }}$ input affects only the $i^{\text {th }}$ output for $i=1,2, \ldots, m$.

Using the bond graph representation, we propose a method for decoupling linear time-varying models. In this study it has been considered that the time-varying parameters of the system are the characteristic functions of the elements of the bond graphs (C,I, R, TF, GY, Se, $S f$ ) and not the topology of the graph.

The following section presents a short theoretical recall of numerical decoupling of LTV models. In the sequel some definitions for the bond graphs models are introduced in the time-varying case. In the third section, the theoretical main result is presented, followed by an application which is well known to the system control community.

\section{PRELIMINARY DEVELOPMENTS}

In this section the methods developed for the decoupling are reviewed.

\section{Decoupling Problem}

The procedure proposed by (Porter 1969) for inputoutput decoupling of linear time-varying systems is recalled. The algorithm is defined for numerical purposes, but it offers a lead over the steps to be followed. 
The differential operator $L=s I+A^{T}$ is defined. It can be applied to the state vector as defined in (3). The operator $s$ is $s=\frac{d}{d t}$.

$$
(L x)(t)=s x(t)+A^{T}(t) x(t), t \in I
$$

If $c_{i}, i=1,2, \ldots, m$ denotes the $i^{\text {th }}$ row of the matrix $C$, then indices $n_{i}$ can be defined as in equation (4).

$$
n_{i}=\min \left\{j \mid B^{T}(t)\left(L^{(j-1)} c_{i}^{T}\right)(t) \neq 0\right\}, t \in I
$$

In case of LTV models the indices $\left\{n_{i} \mid i=1, \ldots, m\right\}$ are time dependent and not necessarily finite.

Using the differential operator and the indices defined above, the matrices $\tilde{A}$ and $\tilde{B}$ are defined like in equations (5) and (6) respectively.

$$
\begin{gathered}
\tilde{A}(t)=\left[\begin{array}{c}
\left(L^{\left(\alpha_{1}-1\right)} c_{1}^{T}\right)^{T}(t) \\
\vdots \\
\left(L^{\left(\alpha_{m}-1\right)} c_{m}^{T}\right)^{T}(t)
\end{array}\right], t \in I \\
\tilde{B}(t)=\tilde{A}(t) B(t), t \in I
\end{gathered}
$$

Property 1: The LTV model defined by (1) is decoupled by a static feedback (2) iff matrix $\tilde{B}(t)$ is non-singular.

The relations (7) and (8) give the expression of matrices $F$ and $G$, where $\Lambda(t)$ is an arbitrary diagonal matrix which may impose a desired behaviour to the decoupled system (Porter 1969).

$$
\begin{gathered}
F(t)=-\tilde{B}^{-1}(t)[\tilde{A}(t) A(t)+(s \tilde{A})(t)], t \in I \\
G(t)=\tilde{B}(t) \Lambda(t), t \in I
\end{gathered}
$$

The steps taken for the decoupling of linear timevarying systems in (Porter 1969) are:

1. Determine the indices $n_{i}$ for each output;

2. Calculate the matrices $\tilde{A}(t)$ and $\tilde{B}(t)$;

3. Verify whether property 1 is true, if not the procedure stops;

4. Calculate the inverse matrix for $\tilde{B}(t)$;

5. Determine matrices $F(t)$ and $G(t)$ according to the relations (7) and (8).

The algorithm can be split into two separeted parts: the first three steps represent the analysis and the last two the synthesis of the decoupling law.

\section{MAIN RESULT}

Even though in (Bertrand 1997), a bond graph approach has been proposed for the decoupling of LTI models, this perspective has it is limitations and it cannot be applied for the linear time-varying models. The algorithm has to be generalized. Even a simple multiplication of two polynomials in $s$, with coefficients variable in time has to be redefined to suite equation (9).

$$
s a=a s+\dot{a}
$$

The non-commutative aspects have to be taken into account. Mason's rule, used for determining the transfer function in a bond graph, does not hold any more for the time-varying systems and therefore a new approach should be used for the non-commutative rings. This rule is called Riegle's rule and it was introduced for calculating the transfer function for the time-varying systems modelled by bond graph in (Achir et al. 2004).

Seeing the aspects involved, the article presents new, more general definitions for the concepts used in system decoupling. In the following subsections the procedure for solving decoupling problem is discussed in the context of linear time-varying models.

\section{System Analysis}

The first step is to enunciate the problem:

Given the system (1), is it possible to determine a regular static feedback control law (2), which decouples the global system?

The answer to this question is given by studying the structure at infinity of the system. In (Descusse and Dion 1982), the case of linear time-invariant systems is studied by means of the structure at infinity.

Theorem 1: (Descusse and Dion 1982) The LTI square system $\Sigma(C, A, B)$ can be decoupled by a static regular control law if and only if the orders of infinite zero are respectively equal to the orders of infinite zero of the row sub-systems $\Sigma\left(c_{i}, A, B\right), i=1, \ldots, m$.

But the interest of this article is with the linear timevarying systems' case. Therefore the definitions for the global orders of infinite zero, the orders of infinite zero for the row sub-systems, as well as the procedure for their computation have to be updated.

The aim of this section is to offer a graphical technique, by means of the bond graph representation to determine whether the system can be decoupled or not.

Definition 1: Given a Smith-McMillan form at infinity $\Phi(s)$ of the transfer matrix $T(s)$ in equation (10), the coefficients $n_{i}^{\prime}$ in decreasing order are the orders at infinite zero of the system. 


$$
\Phi(s)=\left[\begin{array}{cccc}
s^{-n_{1}^{\prime}} & & & 0 \\
& \ddots & & \\
& & s^{-n_{r}^{\prime}} & \\
0 & & & 0
\end{array}\right]
$$

Property 2: (Falb and Wolovich 1967) The orders of infinite zero are equal to the minimal number of derivations of each output variable necessary so that the input variables appear explicitly and independently in the equations.

Definition 3: The order of infinite zero for the row subsystem $\Sigma\left(c_{i}, A, B\right)$ is the integer $n_{i}$, which verifies condition (11).

$$
n_{i}=\min \left\{j \mid B^{T}(t)\left((A(t)+s I)^{j-1} c_{i}^{T}(t)\right) \neq 0\right\}
$$

Property 3: $n_{i}$ is equal to the number of derivations of the output variable $y_{i}(t)$ necessary for the input variables to appear explicitly.

There are some differences between the time invariant definitions of the structural properties of the bond graphs and the time-varying case caused by the fact that the elements of the bond graph $(C, I, R, T F, G Y)$ are time dependent and therefore the definition of gain of a causal path and other aspects concerning the differential equations need to be reviewed from the noncommutative perspective.

Definition 4: The length of a causal path is equal to the number of dynamic elements in integral causality along the path.

Property 4: The order of infinite zero for the row subsystem $\Sigma\left(c_{i}, A, B\right)$ represented by a bond graph model is equal to the length of the shortest causal path between the output detector and the set of input sources.

Property 5: The orders of infinite zero of a global bond graph model is calculated according to equation (12), where $L_{k}$ is the sum of the $k$ shortest different inputoutput causal paths.

$$
\left\{\begin{array}{l}
n_{1}^{\prime}=L_{1} \\
n_{k}^{\prime}=L_{k}-L_{k-1}
\end{array}\right.
$$

According to property 4 and theorem 1 , it is then possible to conclude on the decoupling property of the bond graph model only with a graphical approach.

This algorithm is not very different from the one proposed by (Bertrand 1997) for the LTI models, the definitions have been generalized so that they are adequate to the LTV systems.

\section{Control Synthesis}

Once the indices $n_{i}$ are determined, the next step is to determine the matrices $\tilde{A}(t)$ and $\tilde{B}(t)$, which consists in calculating the vectors $\left(A^{T}+S I\right)^{n_{i}-1} C_{i}^{T}$ and $B^{T}\left(A^{T}+S I\right)^{n_{i}-1} c_{i}^{T}$ respectively. The procedure is similar for determining the two matrices, therefore we focus on $\tilde{A}(t)$, afterwards the differences for calculating $\tilde{B}(t)$ will be pointed out.

For an easier comprehension of the procedure, the graph representation of a bond graph is used, but the technique can be directly used the same way on a bond graph model.

First, the graph associated to the system is rewritten. The state nodes are the derivatives of the state variables and the operators on each edge of the graph are calculated accordingly as in equation (13).

$$
\left\{\begin{array}{l}
s x(t)=A(t) s^{-1}(s x(t))+B(t) u(t) \\
y(t)=C(t) s^{-1}(s x(t))
\end{array}\right.
$$

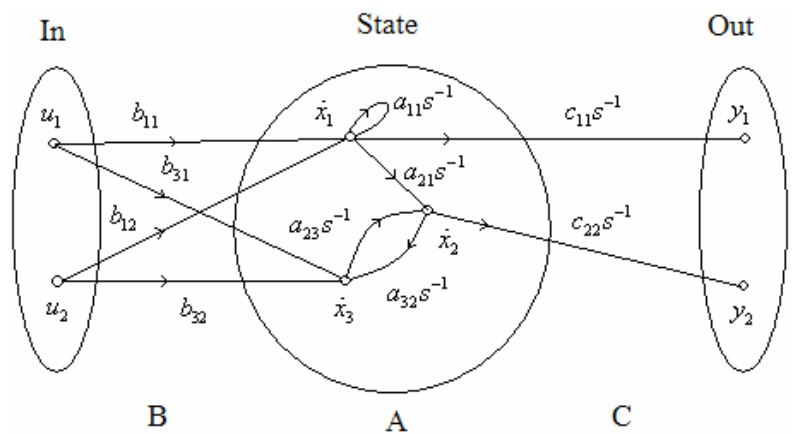

Figure 1. Graph Representation

In figure 1 the sets In, State and Out represent the sets of input sources, state variables and output detectors respectively. The gains of the arcs between the nodes in the sets In and State are equal to the values from the matrix $B(t)$. The gains of the arcs between the nodes in the set State and between the nodes in the sets State and Out are equal to the values from the matrices $A(t)$ and $C(t)$ multiplied by $s^{-1}$.

Property 6: The vectors $\left(A^{T}+S I\right)^{n_{i}-1} c_{i}^{T}$ are determined according to the formula (14), where $p_{i j}$ is the number of paths of length $\left(n_{i}-1\right)$ between the $i^{\text {th }}$ output and the $j^{\text {th }}$ state variable and $G_{k}\left(X_{j}, y_{i}\right)$ is the gain of the path which is calculated according to the relation (15). 


$$
\begin{gathered}
\left(A^{T}+s I\right)^{n_{i}-1} c_{i}^{T}=\left(\sum_{k=1}^{p_{i j}} G_{k}\left(X_{j}, y_{i}\right)\right) X_{j} \\
G_{k}\left(X_{j}, y_{i}\right)=\prod_{r=1}^{\alpha_{i}-1}\left(g_{r}+\gamma_{r} s\right)
\end{gathered}
$$

In equation (15) $g_{r}$ is the gain of the $r^{\text {th }}$ arc along the path and $\gamma_{r}$ is a parameter which is one if the $r^{\text {th }}$ arc has the same node as head and tail and zero otherwise.

Property 6 allows calculating the formal expression of the vectors $\left(A^{T}+S I\right)^{n_{i}-1} c_{i}^{T}$ directly on the bond graph representation using a graphical technique. The expression determined for these vectors after the procedure presented above represents a multiplication of polynomials in $s$ with time-varying coefficients. Therefore some simple transformations should be made according to the commutation relation (9).

For determining the matrix $\tilde{B}(t)$ the only difference which should be added is that instead of using the paths between the state and the output, the paths between the input and the output have to be used. Once the matrices $\tilde{A}(t)$ and $\tilde{B}(t)$ are calculated, matrices $F$ and $G$ are obtained, according to relation (7) and (8) respectively.

The advantages of the bond graph approach can be seen both in the analysis of the decoupling problem and in the control synthesis. In the analysis, the user does not have to perform the tedious work of computing the vectors, until he finds the order of infinite zero. And then verify whether a non-singular matrix is obtained. Only by identifying the causal paths on the bond graph we can determine whether the model can be decoupled or not. In the control law synthesis the matrices $\tilde{A}(t)$ and $\tilde{B}(t)$ can be easily calculated by using the gain of the causal paths.

\section{EXAMPLE}

The example considered in this section is well known. It was used in (Bourlès and Marinescu 1999) and represents a transmission line (Fig. 2).

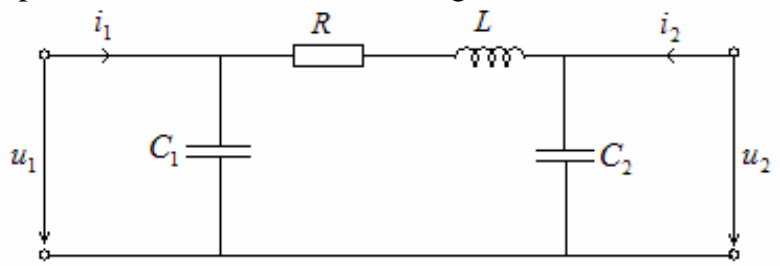

Figure 2. Time-Varying System - Transmission Line

The bond graph model of this system is presented in figure 3 and its state representation is described in equation (16). The system is considered time-varying and the time-dependence is reflected by the values of the bond graph elements $C, I$ and $R$ which are time functions.

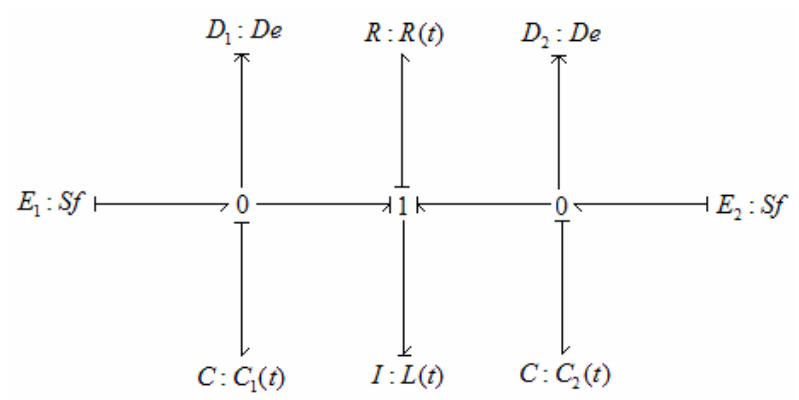

Figure 3. Bond Graph Model

$$
\begin{gathered}
A=\left(\begin{array}{ccc}
-\frac{R(t)}{L(t)} & \frac{1}{C_{1}(t)} & \frac{1}{C_{2}(t)} \\
-\frac{1}{L(t)} & 0 & 0 \\
-\frac{1}{L(t)} & 0 & 0
\end{array}\right) \quad B=\left(\begin{array}{ll}
0 & 0 \\
1 & 0 \\
0 & 1
\end{array}\right) \\
C=\left(\begin{array}{ccc}
0 & \frac{1}{C_{1}(t)} & 0 \\
0 & 0 & \frac{1}{C_{2}(t)}
\end{array}\right) \quad D=\left(\begin{array}{ll}
0 & 0 \\
0 & 0
\end{array}\right)
\end{gathered}
$$

In order to facilitate the comprehension of the procedure, the graph representation of the bond graph is also presented in figure 4 and the discussion is related on the two graphic representations in parallel.

The first step consists in determining the order of infinite zero $n_{i}$ for each sub-system $\Sigma\left(c_{i}, A, B\right)$. The shortest path for $D_{1}$ is $E_{1}-C_{1}-D_{1}$, which has the length 1 and therefore $n_{1}=1$. Analogously the shortest path for the second output $D_{2}$ is $E_{2}-C_{2}-D_{2}$ and $n_{2}=1$.

Secondly the orders of infinite zero of the global system have to be determined. The shortest causal path is $E_{1}-C_{1}-D_{1}$ and has a length of 1 , therefore $L_{1}=n_{1}^{\prime}=1$. The two shortest different causal paths are $E_{1}-C_{1}-D_{1}$ and $E_{2}-C_{2}-D_{2}$, therefore $L_{2}=2$ and $n_{2}^{\prime}=L_{2}-L_{1}=1$.

The orders of the infinite zeros of the row sub-systems are $\{1,1\}$ and the orders of the infinite zeros of the global system are $\{1,1\}$. According to theorem 1 , this model can be decoupled by a regular static law of type (2). 


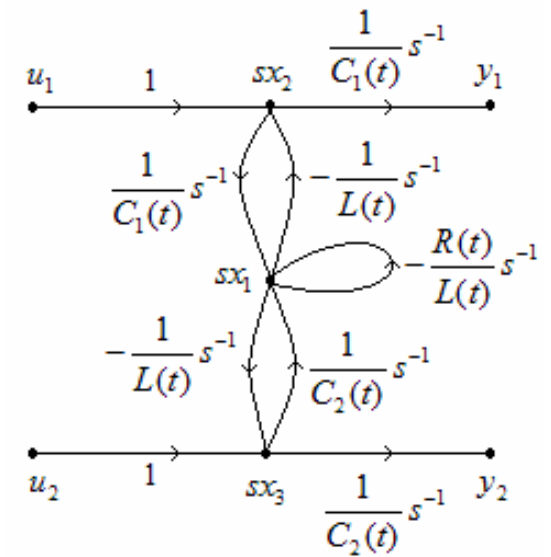

Figure 4. Graph Representation of the system

Once the indices $n_{i}$ are determined one can proceed to the computing of matrices $\tilde{A}(t)$ and $\tilde{B}(t)$. Applying property 6 is straight-forward: for the first output, the causal path gain between $C_{1}-D_{1}$ is $\frac{1}{C_{1}(t)}$; for the second output, the causal path gain between $C_{2}-D_{2}$ is $\frac{1}{C_{2}(t)}$. Matrices defined in relations (17) and (18) are respectively:

$$
\begin{gathered}
\tilde{A}(t)=\left(\begin{array}{ccc}
0 & \frac{1}{C_{1}(t)} & 0 \\
0 & 0 & \frac{1}{C_{2}(t)}
\end{array}\right) \\
\tilde{B}(t)=\left(\begin{array}{cc}
\frac{1}{C_{1}(t)} & 0 \\
0 & \frac{1}{C_{2}(t)}
\end{array}\right)
\end{gathered}
$$

The inverse of matrix $\tilde{B}(t)$ is determined (relation (19) ) and then it remains only to apply relations (7) and (8) to calculate the decoupling matrices $F(t)$ and $G(t)$ (equations (20) and (21).

$$
\begin{gathered}
\tilde{B}^{-1}(t)=\left(\begin{array}{cc}
C_{1}(t) & 0 \\
0 & C_{2}(t)
\end{array}\right) \\
F(t)=\left(\begin{array}{ccc}
\frac{1}{L(t)} & \frac{\dot{C}_{1}(t)}{C_{1}(t)} & 0 \\
\frac{1}{L(t)} & 0 & \frac{\dot{C}_{2}(t)}{C_{2}(t)}
\end{array}\right) \\
G(t)=\tilde{B}(t) I_{2}=\left(\begin{array}{cc}
C_{1}(t) & 0 \\
0 & C_{2}(t)
\end{array}\right)
\end{gathered}
$$

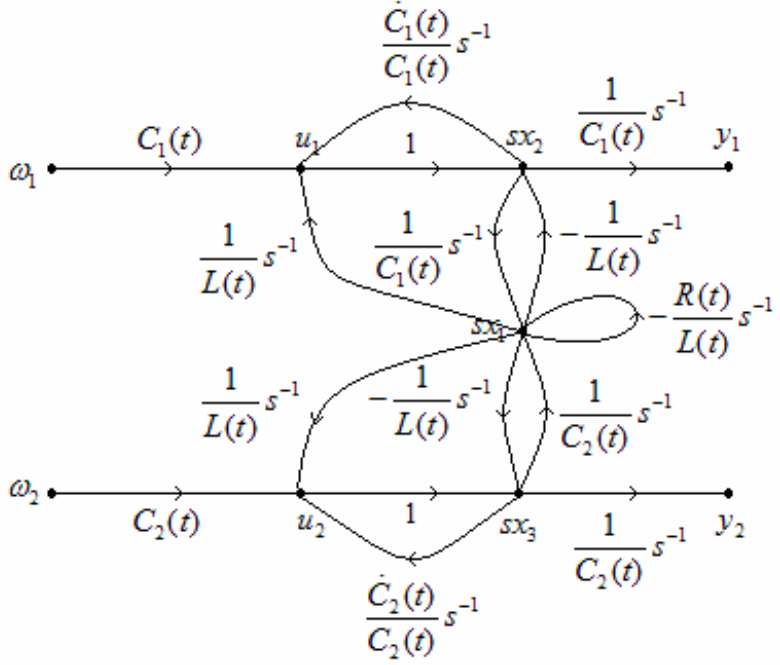

Figure 5. Graph Representation of the decoupled system

The graph of the new decoupled system is presented in figure 5. In order to determine the transfer function of the decoupled system, Riegle's rule (a short recall is presented in the appendix of this article) can be applied. But the result is known here, because this mdel is decoupled

The global transfer matrix can be computed, a diagonal matrix (eq. (22)) is obtained.

$$
T(s)=\left(\begin{array}{cc}
\frac{1}{s} & 0 \\
0 & \frac{1}{s}
\end{array}\right)
$$

Remark 1: After the decoupling, it is possible that certain poles of the system become unobservable and therefore it is important that these poles should be stable. In (Bertrand 1997) a procedure for decoupling LTI models using a pole placement has been developed, using a geometrical approach. The use of $(A, B)-$ invariance for the pole placement has not been so simple to generalize and this issue remains a perspective for our work.

\section{CONCLUSIONS}

In this paper a new graphical technique for the decoupling of linear time-varying systems, based on the bond graph models of the systems was introduced. The procedure can be applied very easily and diminishes the amount of time allocated to the tedious computing of the matrices which are used in decoupling.

Future work concerns the application of these procedures for the decoupling of the nonlinear bond graphs by using the variational bond graph. Also decoupling with stability for the linear time-varying models is an issue which will be considered. 


\section{REFERENCES}

Achir, A., C. Sueur and G. Dauphin-Tanguy. 2004. Ring bond graphs over non-commutative rings. Application to the variational bond graphs. Proceedings of IMAACA.

Bertrand, J.M. 1997. Analyse structurelle et commande par découplage entrée-sortie des modèles bond graphs $(\mathrm{PhD}$ thesis). Ecole Centrale de Lille

Bourlès, H. and B. Marinescu. 1999. Poles and zeros at infinity of linear time-varying systems. IEEE Transactions on Automatic Control, 44:1981-1985.

Commault,C and J.M. Dion 1982. Structure at infinity of linear multivariable systems. A geometric approach. IEEE Transactions on Automatic Control, pages 693-696.

Descusse, J. and J.M. Dion 1982. On the structure at infinity of linear square decoupled systems. IEEE Transactions on Automatic Control, vol. AC-27, No.4, pages 971-974.

Dion, J.M. 1983. Sur la structure a l'infini des systèmes linéaires. (PhD thesis). Institute National Polytechnique de Grenoble.

Falb, P.L. and W.A.Wolovich. 1967. On the decoupling of multivariable systems. Preprints JACC, Philadelphia, pages 791-796.

Pliam, J.O. and E. Bruce Lee. 1998. Ring graphs and gain formulas, an algebraic approach to topology. ISCAS, pages 327-330.

Porter, W. 1969. Decoupling of and inverses for time-varying linear systems. IEEE Transactions on Automatic Control, August 1969:378-380.

\section{APPENDIX}

\section{Riegle's gain formula}

The gain formula over a non-commutative ring was introduced in (Pliam and Lee 1998) for the noncommutative graph, and was extended by (Achir et al. 2004) to the time-varying bond graph models. It can be calculated as in the equation (23), where $P_{(k)}$ is called the path product of the $k^{\text {th }}$ path from input node to exit node and has the expression (24).

$$
\begin{gathered}
T=\sum_{k \in H} P_{(k)} \\
P_{(k)}=A_{n}^{k} \prod_{i=n-1}^{1}\left(1-S_{i}^{k}\right)^{-1} A_{i}^{k}
\end{gathered}
$$

$A_{i}^{k}$ is the $i^{\text {th }}$ edge operator along the $k^{\text {th }}$ path and $S_{i}^{k}$ is the self-gain of the node following the $i^{\text {th }}$ arc with the remaining nodes along the path.

This formula is used to calculate the transfer function for a time-varying system modelled by a bond graph and was introduced for proving directly on the bond graph representation that the decoupling actually takes place. 